\title{
A review and critical assessment of cycling infrastructures across Europe
}

\author{
A. Vassi \& T. Vlastos \\ National Technical University of Athens, Greece
}

\begin{abstract}
Cycling is an old but fast growing component of European cities' mobility planning schemes. Cycling has strategic importance for the mobility management schemes and the sustainable development of European cities. Bicycle infrastructure offers excellent value for money compared with other types of transport. In some cities, the traffic system already offers a large amount of bicycle infrastructures. This paper will be dealing with the categorization of cycling infrastructures across Europe and on its contribution to the mobility management of cities. This study has gathered data concerning the cycling infrastructure across Europe through an online questionnaire, common for all cities. The main benefits and challenges of infrastructures, their effectiveness in mobility management, and their safety performances are presented.
\end{abstract}

Keywords: cycling infrastructure, mobility management plan.

\section{Background}

Cycling is an old but fast growing component of European cities' mobility planning schemes. However, despite the extensive re-introduction of cycling, there is still significant potential for further growth - with some urban areas having gathered valuable experience and others still exploring the field. Cycling has strategic importance for the mobility management schemes and the sustainable development of European cities. This document is an output of the INTERREG IVC CycleCities project and provides a critical overview on cycling infrastructure in European cities. 
CycleCities aims to build and share knowledge and facilitate good practice transfer and experience exchange among European cities on the integration of cycling into urban mobility management schemes. It specifically aims to:

$>$ Exchange experiences and make transferable good practices on mobility management and cycling available to European stakeholders.

$>$ Establish consensus on policies towards sustainable European mobility management schemes.

$>$ Establish a European, multilingual, freely-accessible knowledge and experience base.

$>$ Disseminate field experiences and project results as a means to enhance awareness on the integration of cycling in urban mobility management schemes.

Cyclecities addresses some critical challenges and opportunities for European cities that relate to a number of factors:

Traffic congestion: $30 \%$ of car trips in Europe are under $3 \mathrm{~km}$ and $50 \%$ are under 5km - a 15 minute bike ride (EEA Report No 5/2009 [1]). Reducing car use and increasing cycling will unclog roads and reduce congestion and associated delays, lost working hours and wasted fuel.

Cost reduction: Motorised transport imposes high costs on individuals and society, both directly (road construction and maintenance) and indirectly (casualties, obesity, pollution, congestion, etc.). The European Commission (COM 2009/279 [2]) estimates the external costs of road transport (mostly individual motorised transport) at $2.6 \%$ of GDP. Other studies suggest as much as $4 \%$ and $8 \%$. Shift from car to cycling provides an opportunity for huge cost savings.

Lower carbon footprint: Some 40\% of Europe's CO2 emissions from road transport and $70 \%$ of other pollutants are due to urban traffic. As recognised in EU Communication 2009/279 [3], urban transport accounts for $40 \%$ of CO2 emissions, and 70\% of other air pollution, in particular PM10 and NOx emissions, from transport. Tripling the modal share of cycling would save 5\% of transport $\mathrm{CO} 2$ emissions by 2020 . This would make a significant contribution to mitigating climate change and decreasing dependency on fossil fuels.

Health benefits: Increasing the modal share of cycling enhances physical and mental health. Accidents involving cars are associated with cycling and walking, too. Nevertheless, on balance, the benefits to life expectancy of choosing to cycle are 20 times the injury risks incurred by that choice (WHO, 2000 [4]). Higher proportions of commuter cyclists are correlated with lower risks of casualties. Car drivers are used to the presence of cyclists and are more likely to be cyclists themselves.

Land use: increased uptake of cycling leads to reduced land consumption: 10 bikes can be parked in the space required for one car. One lane of typical road can accommodate 2,000 cars per hour - or 14,000 bikes. Fostering of investment and neighborhood revitalisation: Cycle-friendly cities attract individuals \& businesses investment, encourage neighborhood revitalisation and can improve a city's quality of life and environment. 


\section{Cycling infrastructure overview}

The bicycle is an important and strategic mean of transport in urban areas. In cities, the transport system already offers a large amount of bicycle infrastructures e.g. bicycle lanes with special pavement, bicycle tracks, green corridors, shared spaces - and cycling policies, campaigns and cyclist friendly traffic regulations. The issue of cycling infrastructure should not be encountered alone. Infrastructure building is parallel to a component of a broader group of policies that touch on urban planning and political awareness of citizens. The infrastructure for cycling takes space from the car so affects the traffic organization. Thus, the policy of promoting bicycle belongs to a broader policy of restricting car and decreasing speeds. Since it is not possible to construct infrastructure throughout the city, a policy for cycling could be based on measures and implementations in order to transform neighborhoods in low speed areas. In this case the bicycle will share the space with the other vehicles.

\section{1 "Routes not lanes or tracks"}

Cycle infrastructures are translated in cycle network in the city as a whole. In Prestos' Cycling Policy Guide Infrastructure [5], the definition of cycle networks can be found: "a cycle network is an interconnected set of safe and direct cycling routes covering a given area or city".

Infrastructure for cyclists requires space availability and investment. An integrated, seamless network of cycle routes can greatly improve the attractiveness and safety of cycling. Cycle networks can connect origins and destinations quickly and provide more secure conditions by separating cyclists from motorised traffic. A cycle route must fit closely to the criteria of safety, directness, cohesiveness, comfort and attractiveness.

In many cities, cycle routes begin and end to nowhere. Tracks which are fragmented and scattered certainly could not be described as a network. A continuous and legible cycling network is the desired goal for cities. For a cycling network to attract the widest possible segment of the population, its most fundamental attribute should be "low-stress connectivity", that is, as stressed by Mekuria (2012) [6], "providing routes between people's origins and destinations that do not require cyclists to use links that exceed their tolerance for traffic stress, and that do not involve an undue level of detour".

The concept of connectivity also relates to the cycling network connection with other modes of transport. Coordinating bicycling with public transport is mutually beneficial, enhancing the benefits of both modes and encouraging more bicycling as well as more public transport use (Brons et al. [7], Givoni and Rietveld [8], Hegger [9], Martens [10] and [11], US DOT [12]).

It's not enough for a bicycle network to be connected it also needs to be connected safely. A recent study from Hunter College at the City University of New York (Tuckel [13]), reveals a "greater tendency to ride on more 'secure' street or avenue environments than their cycling counterparts". 


\subsection{Shared economy: shared facilities}

Urban development in recent decades has focused mainly on: more residents, more mobility, more prosperity, etc. Although this method of planning still applies, the needs of urban communities (aging society, affected by the economic crisis) have changed. As urban development, , is a reality for most European cities, the focus will be less and less on further ground cover, as there is (will be) less and less demand, but it will be more on management of the existing urban infrastructures. One of the major components that affect planning is the tendency and need not to own but to barter, lend, trade, gift and swap goods or services. It is called 'collaborative consumption' or 'sharification'. The economy of sharing is a way for individuals, governments and businesses to access goods, space, money, and services in new and less expensive ways. The projection of collaborative consumption in urban space represents ancient and perceivable spatial relationships between populations and mixes different political, administrative institutional and cultural perspectives in an effective scale.

This trend is also affecting cycling infrastructures. It can be found more and more solutions that are based on sharing infrastructures and not constructing new ones. Examples are sharrows, fahradstrasse, shared bus and bike lanes and even the bike sharing systems that are spreading across Europe. This is a result of the economic crisis in some cities, due to the lack of space, or due to value of land in others. It is also because planners, citizens and decision makers understood the importance of calming the traffic, whether in an area around home, schools, or cultural centers, which can be achieved by mixing cars and bicycles, instead of building more infrastructure.

\section{Survey and important results}

\subsection{Survey}

The physical characteristics of the cities vary significantly. Most obviously their population differs greatly. Their natural environment also varies. Some cities are located in coastal areas, others are built in hilly regions and others stand on flat areas. There are also many differences between cities as regards the transport conditions. There are municipalities belonging to strongly car-oriented metropolitan areas. On the other hand cycling has a long tradition in many European cities.

It is very important to understand the features of the cities in which the infrastructure belongs in order to understand the physical and particular characteristics of each case. The first part of the questionnaire included general information concerning the city and its existing cycling infrastructure: e.g. lanes, junctions and off road facilities. In the next section is asked the description of all the cycling infrastructures of the city. Road layouts were attached in order to help people to answer the questionnaire. The next part concerned the description of 3 typical routes: the width of the route, the reasons for choosing the specific route, the main points of interest that are connected by each route the type of areas and 
description of traffic measures that accompanied the construction of the cycle route. The final section refers to the city scale and generally to the planning of cycle routes: parking/BSS, signposting, the possible effects of the network on the city and safety.

The questionnaire set light on the most important issues, the main benefits and challenges of infrastructures faced by the planners.

\subsection{Categorization of results}

There is a lot of experience worldwide concerning cycling infrastructure. This experience in combination with the questionnaire leads us to the following categorization.

\subsubsection{Infrastructure for transport of goods by bicycle and taxi bikes}

This is a big issue in most European cities is transferring goods by bike and taxi-bikes. In almost all the cities, which have a historic, low speed or pedestrianized center, taxi bikes exist for tourist reasons, but also for those who do not have the physical capability to walk. As a mean for tourists to explore cities, it is considered- after walking- the best way to get familiar with a place, to find out the true city, to feel it.

Another aspect of urban cycling is the cycle logistics. Almost $100 \%$ of the transport of goods in cities is done by motorised vehicles, ranging from personal cars to lorries. Light goods are often transported over very short distances by heavy vehicles. Even if the length of trips and the possibility for trip chains is taken into consideration, this still leaves a potential of $25 \%$ of all trips that could be shifted from motorised vehicles towards cycling-related solutions. Post companies can reduce energy and space used in urban freight transport and decrease traffic by replacing unnecessary motorised vehicles with bikes or with cargo bikes for intra-urban delivery.

\subsubsection{Parking}

There is consensus on the need to provide good bike parking for cyclistsespecially secure, sheltered parking to prevent theft and to protect bicycles from difficult weather conditions (Litman [14], Netherlands Ministry of Transport [15], Pucher and Buehler [16]).

The importance of bike parking is obvious, but as Pucher et al. [17] state, few studies have even attempted to measure the impact of bike parking on cycling levels, thus it is not clear to what extent providing parking facilities follows increased cycling levels instead of preceding and encouraging more cycling.

The results of the survey show that a $60 \%$ of the cities in Europe have sheltered bicycle parking. In $85 \%$ of the cities there are unsheltered bicycle parking. The common thing is that parking facilities in case they exist, they are near a public transport station highlighting the importance of the integration of the cycling network with public transport. 


\subsubsection{Public transport and bicycle}

Cycling supports public transport by extending the catchment area of transit stops far beyond walking range and at much lower cost than buses and park-and-ride facilities for cars. Access to public transport helps cyclists to make longer trips than could be possible only by bike use. Transit services also can provide convenient alternatives when cyclists encounter bad weather, difficult topography, gaps in the bikeway network, and mechanical failures. Martens [18] notes the success of the Dutch public transport bicycle system (OV-Fiets), which provides convenient and inexpensive short-term bicycle rentals (using automated smart card technology) for trips from major train stations to the final destinations of travelers, usually near the city center. The evidence of questionnaire confirms that better integration of cycling with public transport leads to more bike and ride trips, and probably to more cycling overall. Bicycle and transit integration is also thought to decrease automobile traffic congestion, help reduce air pollution (by reducing motor vehicle trips), and improve the public image of transit.

Bicycles are accommodated on buses that travel within and between cities, in several different ways. The most used method is to mount a bicycle rack on the front of the bus (Figure 1) (this solution is not permitted by the European legislation for safety reasons). Customers are responsible for loading and securing their bikes on the racks, and the racks can be folded up when they are not in use. Some bus services allow passengers to bring their bicycles on board. However, this method of bicycle accommodation is often restricted to prevent crowding. Bus drivers decide when to allow bicycles on the bus, which tends to be when available bus bike racks are full, after dark, or when bus service is infrequent (bicycles are often allowed on board if the bus is the last bus on the route or if there will be a long wait before the next bus).

A number of rail systems (within and between cities) accommodate bicycles by allowing them inside trains. One method of accommodation is to require cyclists to board designated rail cars and remain with their bikes in specific areas. Some rail cars have special bike racks or hooks where bicyclists can store their bikes. It is common for transit agencies to prohibit bicycle access on train cars during peak travel times. This is done to reduce congestion on the train and to reduce friction in boarding and exiting the train.

\subsubsection{Infrastructure that supports bicycle users}

An important component of the cycling infrastructure is the infrastructure that supports cycling, either directly or indirectly. This includes wholesale businesses (distribution) in bicycles, parts, and accessories, bicycle repair shops and retail shops in bicycles and parts. The questionnaire identified businesses in cities that vary in size and services offered: selling bicycles, repair of bicycles, trade of bicycle parts, bicycle manufacturing. There are also services aiming to "facilitating" the cyclists as hotels that provide facilities for cycling tourists, informational signage of a city and ramps and elevators installed to overcome steep slopes (Figure 2). 


\subsubsection{Infrastructure for tourism}

Leisure cycling has an important role to play in preserving the cycle habit. Cycling holidays accounts an important percentage of total holiday trips in some European countries and it is predicted to increase within the next decade.

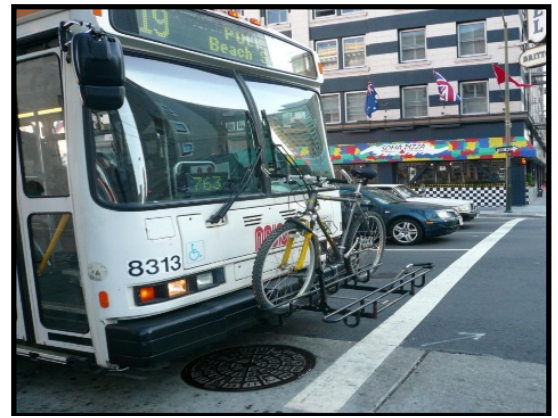

Figure 1: Bicycles on buses (San Francisco) (source: personal archive).

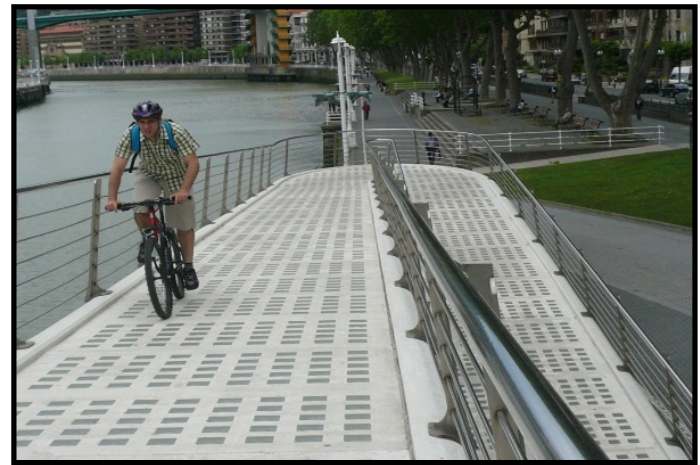

Figure 2: $\quad$ Ramp for bicycles (source: personal archive).

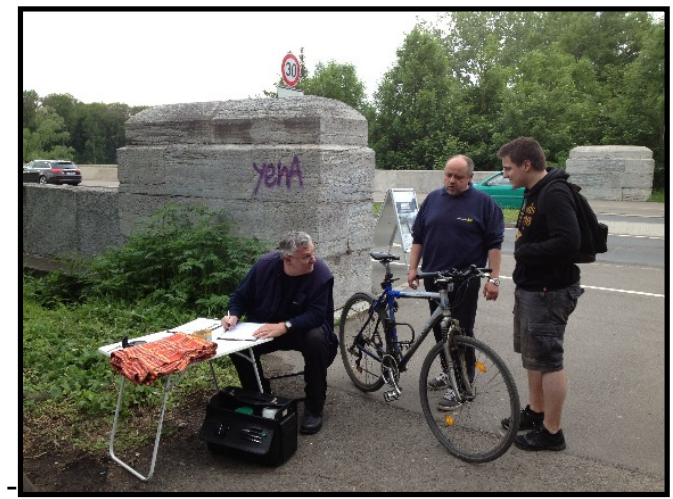

Figure 3: Bicycle registration in Leipzig, Germany (source: personal archive). 
Holiday cycling is even more popular. Cycle tourism is developing rapidly across Europe as it is shown by European commission's 'EuroVelo' TransEuropean cycle route network scheme. There are several types of infrastructure that promote cycling tourism such as the cycling routes along the rivers and those which connect countries, like the routes planned by the European project CYRONMED - Cycle Route Network of the Mediterranean, financed by INTERREG III.

\subsubsection{Infrastructure for information}

The information you can receive concerning cycling in a city is very important. Maps, applications, navigators, blogs, and press they all contribute to make cycling more popular. The online maps (e.g. http://www.sustrans.org.uk/ncn/map) and urban cycling blogs (e.g. http://www.copenhagenize.com/) can increase the number of cyclists in a city by providing information enhancing the feeling of safety. Through the questionnaire the importance of applications with maps that navigate you through cycle routes (e.g. Radplaner) was highlighted, especially in places that are visited for a first time by the user.

\subsubsection{Institutional support}

In many European cities there are bicycle offices in municipalities, regions or even in ministries. The questionnaire revealed that the institutional support for cycling plays a very positive role: planning and maintaining the cycling network, improving the education and public awareness, and generally promoting the cycling culture. A typical example is the bicycle office in the municipality of Leipzig, Germany, where it is involved in all the above mentioned activities. Additionally in order to reduce bicycle thefts, it registers the bicycles and their owners (Figure 3).

\subsection{Bicycle challenges and potentials}

\subsubsection{Segregated vs. non segregated infrastructure}

The survey shows the variety of bike infrastructure in Europe: cycle tacks, cycle lanes, advisory lanes, sharrows etc. In countries that are considered pioneers in the field of cycling, fewer types of infrastructure can be detected which are based on the use of existing roads either segregated or not. In cities that have no such bicycle culture, infrastructure that separates the bicycle movement from the car is preferred, even if the construction of such infrastructure is costly and it is obvious that in the intersections there are safety problems because drivers are not conscious of the existence of cyclists if they are on the sidewalk or on segregated tracks.

Research shows that the biggest dilemma in relation to infrastructure planning is choosing segregated or non-segregated infrastructure. In literature, there are several reports on evaluation bicycle networks (Aultman-Hall et al. [19], Aultman-Hall and Adams [20], Vlastos [21] for a broad overview of relevant research). The rule usually verified with respect to the behavior of cyclists is 
that proposed by Wilkinson [22], who has divided cyclists into two categories: those who typically do not use the proprietary infrastructure for cycling, not hesitating to follow roads of high risk (high loads, high speeds), and those who choose them systematically.

The rise of non-segregated infrastructures is obvious. Sharrows are the shared lane markings, a new pavement marking that is placed in a lane shared by cars and cyclists. The marking is designed to encourage cyclists to ride at the safest position and to alert motorists to the possible presence of cyclists. Furth and Dulaski [23] point out that without a clear cycling zone being designated, cyclists and motorists have to negotiate for the boundary of the bicycle zone. Fahrradstrasse are the roads mainly addressed to cyclists. Cars are allowed to circulate in fahrradstrasse, but they should give priority to bicycles. In both cases the importance is given to not constructing a new infrastructure, but to use the existing one and to calm the traffic.

Clearly there are pros and cons to segregated and non-segregated infrastructure, but as it is highlighted by the survey, the efficiency of the infrastructure depends on the measure that accompany its establishment: when a non-segregated network is established in a city which is not familiar with cycling, the traffic measures that are applied in the wider area are the key to success. These measures can be low speed zones with the use of sharrows and fahrradstrasse solutions.

They are evidences of the tendency to share, even the component of space which reveal the willingness to create places friendlier.

\subsubsection{Bike sharing systems}

Bike sharing systems lie at the core of urban mobility management strategies. They are critical components of current policies and practices to address transport and environmental challenges as part of wider urban mobility management strategies. The key objective of these systems is to provide free or low cost access to bicycles for short distance trips in urban areas as an alternative option to private car use therefore reducing air pollution, noise levels and traffic congestion. Bike sharing is also linked to public transport either as an alternative transport mode or as a short distance ('last mile') solution connecting commuters to public transport hubs.

Table 1: Proportion of municipality area covered by BSS.

\begin{tabular}{|l|c|}
\hline \multicolumn{1}{|c|}{ Proportion of covered area by BSS } & $\mathbf{\%}$ \\
\hline $10-30 \%$ & $29 \%$ \\
\hline$<10 \%$ & $21 \%$ \\
\hline $31-50 \%$ & $18 \%$ \\
\hline$>50 \%$ & $16 \%$ \\
\hline Spans across several municipalities & $16 \%$ \\
\hline Sum of responses included in the analysis & $\mathbf{1 0 0 \%}$ \\
\hline
\end{tabular}




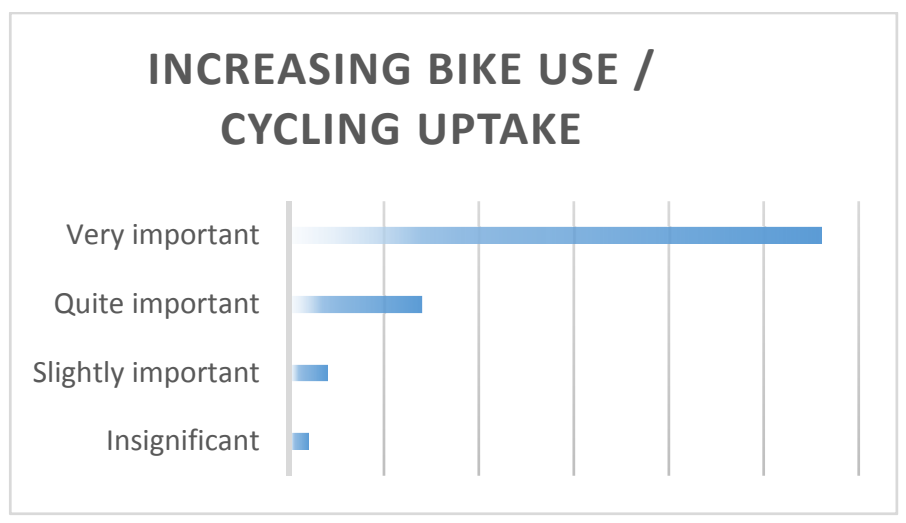

Figure 4: Cycling uptake.

In this context, a 'bicycle sharing system', 'bike sharing system' or 'bike sharing scheme', can be defined as any fully automated, self-service network of bicycles that is available to individuals on a short term basis as a means of short distance transportation in urban areas. Bike sharing systems can be categorized based on financing (public, private, or public-private partnership), ownership, operator and operational model, scale and range.

According to the BSS cases further investigated, 21\% of the BSS in Europe cover less than $10 \%$ of the whole area of the municipality where it operates and $16 \%$ of the systems cover more than $50 \%$ of the area (Table 1 ).

Bike sharing systems are considered able to attract new cyclists (Figure 4), and it is also widely believed that they reduce traffic congestion. It is considered very promising that $74 \%$ of those involved in BSS (operators, companies) consider that the BSS is going to expand.

\section{Conclusions}

The research conducted explored and highlighted the pillars of a cycling city. The results concern the way to build an optimum environment for cycling. Each city has its own needs, its own culture and its own potential to grow cycling culture. The requirements of a cycle network differentiate a lot between cities, but a variety of solutions is provided in order to establish a complete, safe and integrated cycling network. Each city has different geographical, urban and traffic characteristics. A lot of factors influence the effects of cycling infrastructures. Land use planning, car parking policies, car free zones and speed limits are some of the factors that can strengthen or weaken the effects of infrastructure.

Culture, and habit tend to increase cycling in cities with high levels of cycling but also they decrease cycling, especially among non-cyclists, in cities with low levels of cycling. Non- cyclists in bike-oriented cities can respond differently to infrastructures than non-cyclists in cities with little cycling. Non-cyclists who are surrounded by other cyclists it is more likely to start to use bicycle and thus more responsive to infrastructure establishments. Thus, the same infrastructure 
provision, program, or policy might have different impacts on cycling in different cities. Each solution must be "tailor made" for each city. Any type of infrastructure in the proper environment can make people think that cycling is a good option.

In recent years great significance is given to shared infrastructures: shared space, bike sharing systems, sharrows, fahrradstrasse, perspective which reduces infrastructure costs, gives space to pedestrians, lowers speeds in town and therefore make it more humane. We could say that there is a world leading tendency not "equip the warrior", if we describe the cyclists as a warrior in the urban environment, but to "improve the equipment". It is a sign that we passed from the policy of protecting the bicycle to more decisive policies which have as pillars the pedestrians and the cyclists. The new policies are targeting at transforming the city into something more civilized and mild, after all cities are places for people. They need human scale constructions, policies, and measures to cultivate social relationships.

\section{References}

[1] EEA Report No 5/2009.

[2] COM 2009/279, http://eur-lex.europa.eu/LexUriServ/LexUriServ.do?uri= COM:2009:0279:FIN:EN:PDF

[3] EU Communication 2009/279, http://ec.europa.eu/transport/themes/ strategies/doc/2009_future_of_transport/2009_comm_future_of_transport policy_en.pdf

[4] The world health report 2000, http://www.who.int/whr/2000/en/whr00 en.pdf?ua=1

[5] Prestos' Cycling Policy Guide Infrastructure, http://www.polisnetwork.eu/ uploads/Modules/PublicDocuments/presto_cycling-policy-guide-generalframework_english.pdf

[6] Mekuria, M., Low-stress bicycling and network connectivity, Mineta Transportation Institute, Report 11-19, 2012.

[7] Brons, M., M. Givoni, \& P. Rietvield. Access to railway stations and its potential in increasing rail use. Transportation Research, pp. 136-149, 2009.

[8] Givoni, M., and P. Rietveld. The access journal to the railway station and its role in passengers' satisfaction with rail travel. Transport Policy 14, pp. 357-365, 2007.

[9] Hegger, R., Public transport and cycling: living apart or together. Public Transport International 2, 38-41, 2007.

[10] Martens, K., The bicycle as a feedering mode: Experiences from three European countries. Transportation Research Part D, pp. 281-294, 2004.

[11] Martens, K. Promoting bike and ride: The Dutch experience. Transportation Research Part A, pp. 326-338, 2007.

[12] US DOT, Bicycles and transit: A partnership that works. Washington, DC: US Department of Transportation, Federal Transit Administration, 1998.

[13] Tuckel, 2014, http://silo-public.hunter.cuny.edu/62eaab1 fad6c75d37293d2 f2f6504a15adacd5c6/Cycling_Study_January_2014.pdf 
[14] Litman, T., 2009. Bicycle parking, storage, and changing facilities, http://www.vtpi.org/tdm/tdm85.htm

[15] Netherlands Ministry of Transport, 2009. Cycling in the Netherlands. Ministerie van Verkeer en Waterstaat, The Hague. http://www.fietsberaad.nl/library/repository/bestanden/CyclingintheNether lands2009.pdf

[16] Pucher, J., Buehler, R., Making cycling irresistible: Lessons from the Netherlands, Denmark, and Germany. Transport Reviews 28(4), 495-528, 2008.

[17] Pucher, J., Dill, J., Handy, S., Infrastructure, programs, and policies to increase bicycling: An international review. Preventive Medicine 50, pp. 106-125, 2010.

[18] Martens, K. Promoting bike and ride: The Dutch experience. Transportation Research Part A, pp. 326-338, 2007.

[19] Aultman-Hall, L., Hall, F.L. and Baetz, B.B., Analysis of Bicycle Commuter Routes Using Geographical Information Systems: Implications for Bicycle Planning. Transportation Research Record 1578, pp. 102-110, 1997.

[20] Aultman-Hall, L. and Adams, M.F.Jr., Sidewalk Bicycling Safety Issues. Transportation Research Record 1636, pp. 71-76, 1998.

[21] Vlastos, T., Bicycle and environment: Survey on the social, spatial, traffic and institutional conditions for the integration of on sustainable mobility policies in Greece. Research "Pythagoras" first report results. National Technical University of Athens, School of Rural and Surveying Engineering, 2005.

[22] Wilkinson, W.C., Selecting Roadway Design Treatments to accommodate bicycles. FHWA, US Department of Transportation, 1994.

[23] Furth, P., Dulaski, D., More than Sharrows: Lane-Within-a-Lane Bicycle Priority Treatments in Three US Cities. Transportation Research Board Business Office, 2011. 